\title{
ON COMPUTING LEAST ORDER FAULT DETECTORS USING RATIONAL NULLSPACE BASES
}

\author{
A. Varga \\ ${ }^{*}$ German Aerospace Center, DLR - Oberpfaffenhofen \\ Institute of Robotics and Mechatronics \\ D-82234 Wessling, Germany \\ Andras.Varga@dlr.de
}

\begin{abstract}
We propose a numerically reliable computational approach to design least order fault detectors using descriptor system techniques. This approach is based on a new numerically stable algorithm to compute least order rational nullspace bases of rational matrices. The main computation in this algorithm is the orthogonal reduction of the system pencil matrix to a Kronecker-like form. The proposed approach can be combined with coprime factorization techniques to determine stable rational bases. Least order fault detectors can be determined by selecting an appropriate linear combination of basis vectors by eliminating non-essential dynamics. The proposed approach is applicable to both standard and descriptor system descriptions.
\end{abstract}

Keywords: Fault detection, rational matrices, minimal rational basis.

\section{INTRODUCTION}

In the model based fault diagnosis, the fault detection task is achieved by detecting discrepancies between the outputs of the monitored plant and the predictions obtained with a mathematical model. These discrepancies - called also residuals - are indications of faults and are produced by special devices called residual generators. From a system theoretic point of view, the residual generators are physically realizable systems having as inputs the measured outputs and the control inputs of the monitored system, and as output the generated residual. The residual generators are usually implemented as parts of control algorithms or as independent monitoring procedures.

A residual generator has at least two basic functions: (1) generating zero residuals in the fault-free case; (2) generating nonzero residuals when any fault occurs in the system. A more advanced functionality, like fault isolation (i.e., exact location of faults) can be often achieved by designing a bank of fault detectors (Gertler, 1998) or by direct design of fault isolation filters (Varga, 2002). For an efficient implementation and operation of a residual generator (or a bank of such devices), it is generally desirable to keep the order of the fault detector(s) as low as possible. Thus, the design of fault detector with possible least dynamical order is an important aspect from practical point of view.

Although there are many approaches proposed in the literature to design fault detectors (Chen and Patton, 1999; Patton and Hou, 1998; Hou, 2000), only very few of them address explicitly the design of least order detectors. In what follows we restrict ourselves to comment the works (Frisk, 2000; Frisk and Nyberg, 2001), where a design approach of least order detectors is proposed by using polynomial matrix manipulations.

The main computational problem in (Frisk and Nyberg, 2001) is the numerical computation of a minimal polynomial basis for the left nullspace of a certain rational matrix. The method advocated to be used is based on a polynomial echelon form (Kailath, 1980). 
However, this method, as well as similar polynomial manipulation based approaches, contrary to what is said in (Frisk and Nyberg, 2001), are in general numerically unstable. Even the numerically more reliable algorithm of Beelen (1987), implemented in the SLICOT library (Benner et al., 1999), has limitations from numerical point of view, because it involves matrix multiplications and inversions. Although there exist reliable algorithms for many basic polynomial computations, there are two basic limitations for the usefulness of the polynomial approach to solve large order problems.

The first limitation is the intrinsic ill-conditioning of polynomial representations because of possible extremely wide range of polynomial coefficients. It is not uncommon to arrive to polynomial models for which the range of magnitudes of the coefficients exceeds the intervals $\left(\varepsilon_{M}, 1\right)$, or $\left(1,1 / \varepsilon_{M}\right)$, or both of them, where $\varepsilon_{M}$ is the relative machine precision (e.g., $\varepsilon_{M} \approx 10^{-16}$ for double precision computations on many machines). For such models, applying any algorithm (including numerically stable ones) can lead to a complete loss of accuracy, thus to a complete failure.

The second limitation, pointed out by Van Dooren (1981), is that many algorithms based on polynomial manipulations are numerically unstable. The reason for that is simple: typical operations like choosing pivots are determined by powers of the polynomial indeterminate rather than by the numerical values of coefficients. Thus, algorithms to compute minimal polynomial bases (e.g., algorithms based on the Hermite normal form or on the polynomial echelon form (Kailath, 1980)), frequently lead to numerical instability. Therefore, the computed results for large order systems tend to be very inaccurate.

Avoiding the above mentioned difficulties was our main motivation to investigate alternative state-space methods to compute least order nullspaces of rational matrices. Our approach to design least order detectors (see Section 2) is based on a new numerically stable algorithm to compute least order rational nullspace bases of rational matrices (see Section 3 ). The main computation in this algorithm is the orthogonal reduction of the system pencil matrix to a Kroneckerlike form, which allows to obtain, practically without any additional computation, a least order rational nullspace basis. This approach can be combined with coprime factorization techniques to determine stable rational bases. Least order fault detectors can be obtained by selecting an appropriate linear combination of the basis vectors by eliminating non-essential dynamics (see Section 4). The proposed overall approach is applicable to both standard or descriptor systems and represents a numerically reliable alternative to the polynomial approach used in (Frisk, 2000).

\section{DESIGN OF LEAST ORDER DETECTORS}

Consider the linear time-invariant system described by the input-output relations

$$
\mathbf{y}(\lambda)=G_{p}(\lambda) \mathbf{u}(\lambda)+G_{f}(\lambda) \mathbf{f}(\lambda)+G_{d}(\lambda) \mathbf{d}(\lambda),
$$

where $\mathbf{y}(\lambda), \mathbf{u}(\lambda), \mathbf{f}(\lambda)$, and $\mathbf{d}(\lambda)$ are Laplace- or Z-transformed vectors of the $p$-dimensional system output vector $y(t), m$-dimensional plant input vector $u(t), q$-dimensional fault signal vector $f(t)$, and $r$ dimensional disturbance vector $d(t)$, respectively, and where $G_{p}(\lambda), G_{f}(\lambda)$ and $G_{d}(\lambda)$ are the transferfunction matrices (TFMs) from the plant inputs to outputs, fault signals to outputs, and disturbances to outputs, respectively. According to the system type, $\lambda=s$ in the case of a continuous-time system or $\lambda=z$ in the case of a discrete-time system.

A linear residual generator (or detector) of least dynamical order is sought having the general form

$$
\mathbf{r}(\lambda)=R(\lambda)\left[\begin{array}{l}
\mathbf{y}(\lambda) \\
\mathbf{u}(\lambda)
\end{array}\right]
$$

such that: $(i) r(t)=0$ when $f(t)=0$; and $(i i)$ $r(t) \neq 0$ when $f_{i}(t) \neq 0$, for $i=1, \ldots, q$. Besides the requirement that the TFM of the detector $R(\lambda)$ has least possible McMillan degree, it is also necessary, for physical realizability, that $R(\lambda)$ is a proper and stable TFM. As detector, we can always choose $R(\lambda)$ as a rational row vector.

Transcribing the condition $(i)$, we get

$$
R(\lambda) G(\lambda)=0
$$

where

$$
G(\lambda)=\left[\begin{array}{cc}
G_{p}(\lambda) & G_{d}(\lambda) \\
I_{m} & 0
\end{array}\right]
$$

while the condition $(i i)$ requires

$$
R(\lambda)\left[\begin{array}{c}
G_{f}^{(i)}(\lambda) \\
0
\end{array}\right] \neq 0, \quad i=1, \ldots, q
$$

where $G_{f}^{(i)}(\lambda)$ is the $i$-th column of $G_{f}(\lambda)$.

From (2) it appears that $R(\lambda)$ is a left annihilator of $G(\lambda)$, thus one possibility to determine $R(\lambda)$ is to compute first a left minimal basis $N_{L}(\lambda)$ for $\mathcal{N}_{L}(G(\lambda))$, the left nullspace of $G(\lambda)$, and then to build a rational and stable detector as $R(\lambda)=$ $X(\lambda) N_{L}(\lambda)$, representing a linear combination of the rows of $N_{L}(\lambda)$, such that conditions (4) are fulfilled. This approach has been successfully employed by Frisk and Nyberg (2001) to obtain, in several particular cases, least order detectors from computed polynomial bases. We show that a similar approach is possible starting from a computed rational basis, with the obvious advantage of using reliable numerical techniques based on state-space computations. 
The following procedure summarizes our approach for designing a least order detector $R(\lambda)$ :

1. Compute a least McMillan degree basis $N_{L}(\lambda)$ for the left nullspace of $G(\lambda)$ defined in (3).

2. Find a partition of $N_{L}(\lambda)$ as

$$
N_{L}(\lambda)=\left[\begin{array}{l}
N_{L, 1}(\lambda) \\
N_{L, 2}(\lambda)
\end{array}\right]
$$

such that

$$
N_{L, 1}(\lambda)\left[\begin{array}{c}
G_{f}^{(i)}(\lambda) \\
0
\end{array}\right] \neq 0, \quad i=1, \ldots, q
$$

If $N_{L, 1}(\lambda)$ is empty, STOP (no solution exists).

3. Determine a rational matrix $X(\lambda)$ such that

$$
\widetilde{R}(\lambda):=N_{L, 1}(\lambda)+X(\lambda) N_{L, 2}(\lambda)
$$

has the least possible McMillan degree.

4. Define $R(\lambda)$ as the numerator factor of the stable left coprime factorization $\widetilde{R}(\lambda)=M^{-1}(\lambda) R(\lambda)$.

In what follows, we describe in details reliable numerical methods to perform Steps 1 and 3 of the above procedure.

\section{COMPUTATION OF LEAST ORDER RATIONAL NULLSPACE BASES}

In this section we propose a computational approach to determine least order rational nullspace bases of a rational matrix. Besides applications in fault detection, the nullspace computation has many system theoretical applications in model matching, observer design, system inversion (Wang and Davison, 1973; Forney, 1975). A numerically reliable algorithm to compute minimal polynomial bases using state-space techniques has been developed by Beelen (1987) starting from the ideas of Forney (1975). However, the computation of least order rational bases has been apparently not considered yet in the literature.

For convenience, we present a method for the computation of a right nullspace basis of a rational matrix $G(\lambda)$. To compute a left nullspace basis of $G(\lambda)$ (as necessary in the previous section for $G(\lambda)$ defined in (3)), the same approach can be applied to the transposed matrix $G^{T}(\lambda)$.

We assume that $G(\lambda)$ is a $p \times m$ rational matrix of rank $r$, having an observable descriptor system representation $(A-\lambda E, B, C, D)$ satisfying

$$
G(\lambda)=C(\lambda E-A)^{-1} B+D
$$

We consider the problem to compute a $m \times(m-$ $r$ ) rational matrix $N(\lambda)$ whose columns represents a basis for the right nullspace of $G(\lambda)$. Thus $N(\lambda)$ must satisfy

$$
G(\lambda) N(\lambda)=0
$$

Our method exploits the simple fact (Verghese et al., 1979) that $N(\lambda)$ is a nullspace basis of $G(\lambda)$ iff $\left[\begin{array}{l}M(\lambda) \\ N(\lambda)\end{array}\right]$ is a nullspace basis of the system matrix

$$
S(\lambda)=\left[\begin{array}{cc}
A-\lambda E & B \\
C & D
\end{array}\right] .
$$

Thus to compute $N(\lambda)$ we can determine equivalently a nullspace basis $Y(\lambda)$ for $S(\lambda)$ and then $N(\lambda)$ simply results as $N(\lambda)=\left[\begin{array}{ll}0 & I_{m}\end{array}\right] Y(\lambda) . N(\lambda)$ can be computed (see bellow) by employing linear pencil reduction algorithms based on orthogonal transformations. Bases with special properties (e.g., stable) can be obtained by postprocessing $N(\lambda)$, using the fact that if $N(\lambda)=\tilde{N}(\lambda) \widetilde{M}^{-1}(\lambda)$ is a right coprime factorization (RCF), then the numerator matrix $\tilde{N}(\lambda)$ can be equally employed as nullspace basis of $G(\lambda)$.

The main advantage of this approach is that the computation of the nullspace can entirely be done by manipulating state space matrices instead of manipulating polynomial models. The resulting nullspace is obtained in a descriptor system representation which can be immediately used in applications. In what follows we describe in detail our approach.

Let $Q$ and $Z$ be orthogonal matrices (for instance, determined by using the algorithms of (Beelen, 1987; Varga, 1996)) such that the transformed pencil $\widetilde{S}(\lambda):=$ $Q S(\lambda) Z$ is in the Kronecker-like staircase form

$$
\widetilde{S}(\lambda)=\left[\begin{array}{c|cc}
B_{r} & A_{r}-\lambda E_{r} & A_{r, x}-\lambda E_{r, x} \\
\hline 0 & 0 & A_{r e g, c}-\lambda E_{r e g, c}
\end{array}\right]
$$

where the descriptor pair $\left(B_{r}, A_{r}-\lambda E_{r}\right)$ is controllable, $E_{r}$ is non-singular, and $A_{r e g, c}-\lambda E_{r e g, c}$ has full column rank excepting possibly a finite set of values of $\lambda$ (i.e, the invariant zeros of $S(\lambda)$ ). It follows that we can choose the nullspace $\widetilde{Y}(\lambda)$ of $\widetilde{S}(\lambda)$ in the form

$$
\tilde{Y}(\lambda)=\left[\begin{array}{c}
I \\
\left(\lambda E_{r}-A_{r}\right)^{-1} B_{r} \\
0
\end{array}\right]
$$

Then the nullspace of $G(\lambda)$ is

$$
N(\lambda)=\left[\begin{array}{ll}
0 & I_{m}
\end{array}\right] Z \tilde{Y}(\lambda)
$$

and if we partition $\left[\begin{array}{ll}0 & I_{m}\end{array}\right] Z=\left[\begin{array}{lll}D_{r} & C_{r} & C_{r, x}\end{array}\right]$ in accordance with the row partition of $\widetilde{Y}(\lambda)$, we obtain

$$
N(\lambda)=C_{r}\left(\lambda E_{r}-A_{r}\right)^{-1} B_{r}+D_{r}
$$

Thus, $\left(A_{r}-\lambda E_{r}, B_{r}, C_{r}, D_{r}\right)$, with $E_{r}$ nonsingular, is a descriptor system representation for $N(\lambda)$. Note that, to obtain this nullspace basis, we performed exclusively orthogonal transformations on the system matrices. We can prove that all computed matrices are exact for a slightly perturbed original system. It follows that the algorithm to compute the nullspace basis is numerically stable. 
We can show that the rational basis above has actually the least possible McMillan degree. Consider for this the detailed structure of the full row rank subpencil $\left[B_{r} \mid A_{r}-\lambda E_{r}\right]$. We can assume that this subpencil is in the following controllability staircase form

$\left[\begin{array}{c|c|c|c}A_{1,0} & A_{1,1}-\lambda E_{1,1} & \cdots & A_{1, \ell}-\lambda E_{1, \ell} \\ \hline & A_{2,1} & \ddots & \vdots \\ \hline & & \ddots & A_{\ell-1, \ell}-\lambda A_{\ell, \ell} \\ \hline & & & A_{\ell+1, \ell}\end{array}\right]$

where $A_{i+1, i} \in \mathbb{R}^{\nu_{i} \times \nu_{i-1}}$, with $\nu_{0}:=m-r, \nu_{\ell+1}=$ 0 , are full row rank matrices, for $i=0, \ldots, \ell+1$. Note that this form is obtained by using the pencil reduction algorithms described in (Beelen, 1987; Varga, 1996). It follows that the order of the realization (7) is

$$
n_{r}:=\sum_{i=1}^{\ell} \nu_{i}
$$

and we show below that this order is equal to the least possible one.

Theorem 1. The rational matrix $N(\lambda)$ in (7) is a rational nullspace basis of $G(\lambda)$ of least McMillan order.

Proof. Using the staircase form (8), it is shown in (Beelen, 1987) that a minimal polynomial basis can be computed by selecting $\nu_{i-1}-\nu_{i}$ polynomial basis vectors of degree $i-1$, for $i=1, \ldots, \ell+1$. This basis can be used to construct a minimal rational basis by making each column proper with appropriate order denominators. The least order of such a basis is

$$
\bar{n}_{r}=\sum_{i=1}^{\ell+1}\left(\nu_{i-1}-\nu_{i}\right)(i-1)
$$

But this is exactly $n_{r}$, since

$$
\begin{aligned}
\bar{n}_{r} & =\sum_{i=1}^{\ell+1} \nu_{i-1}(i-1)-\sum_{i=1}^{\ell+1} \nu_{i}(i-1) \\
& =\sum_{i=1}^{\ell} \nu_{i} i-\sum_{i=1}^{\ell} \nu_{i}(i-1)=\sum_{i=1}^{\ell} \nu_{i}
\end{aligned}
$$

To finish the proof, we need to show additionally that the realization (7) is minimal. The pair $\left(B_{r}, A_{r}-\lambda E_{r}\right)$ is controllable, by the construction of the Kroneckerlike form (6). To show it is also observable, observe that

$$
\begin{aligned}
{\left[\begin{array}{cc}
Q & 0 \\
0 & I_{m}
\end{array}\right] } & {\left[\begin{array}{cc}
A-\lambda E & B \\
C & D \\
0 & I_{m}
\end{array}\right] Z=} \\
& {\left[\begin{array}{ccc}
B_{r} & A_{r}-\lambda E_{r} & A_{r, x}-\lambda E_{r, x} \\
0 & 0 & A_{r e g, c}-\lambda E_{r e g, c} \\
D_{r} & C_{r} & C_{r e g, c}
\end{array}\right] }
\end{aligned}
$$

has full column rank, excepting those values of $\lambda$ which belong to the unobservable set of eigenvalues of $A-\lambda E$. Since these eigenvalues appears in the subpencil $A_{r e g, c}-\lambda E_{r e g, c}$ (being part of invariant zeros), each of the first two block columns of the second matrix must have full column rank. Therefore, we have that

$$
\operatorname{rank}\left[\begin{array}{c}
A_{r}-\lambda E_{r} \\
C_{r}
\end{array}\right]=n_{r}
$$

and thus the pair $\left(A_{r}-\lambda E_{r}, C_{r}\right)$ is observable.

The TFM $N(\lambda)$ in (7) representing the nullspace basis of $G(\lambda)$ is in general not stable. To compute a stable rational basis, we can replace $N(\lambda)$ by $\widetilde{N}(\lambda)$ determined from a stable RCF $N(\lambda)=\widetilde{N}(\lambda) \widetilde{M}^{-1}(\lambda)$ in the form

$$
\left[\begin{array}{c}
\widetilde{N}(\lambda) \\
\widetilde{M}(\lambda)
\end{array}\right]=\left[\begin{array}{c|c}
A_{r}+B_{r} F_{r}-\lambda E_{r} & B_{r} \\
\hline C_{r}+D_{r} F_{r} & D_{r} \\
F_{r} & I
\end{array}\right]
$$

where $F_{r}$ is determined such that all generalized eigenvalues of the pair $\left(A_{r}+B_{r} F_{r}, E_{r}\right)$ are stable. For this purpose, recursive coprime factorization techniques, as those proposed in (Varga, 1998), can be used. The dual of this approach can be employed at Step 4 of the proposed procedure in Section 2 to obtain a stable detector.

\section{DETECTOR ORDER REDUCTION}

In the procedure in Section 2 we have to solve at Step 3 the following problem: given the partitioning of rows of the left nullspace basis $N_{L}(\lambda)$ as $N_{L, 1}(\lambda)$ and $N_{L, 2}(\lambda)$, determine a rational matrix $X(\lambda)$ such that $N_{L, 1}(\lambda)+X(\lambda) N_{L, 2}(\lambda)$ has least McMillan degree. In a dual formulation we have the problem: for $N_{1}(\lambda):=N_{L, 1}^{T}(\lambda)$ and $N_{2}(\lambda):=N_{L, 2}^{T}(\lambda)$, determine the rational matrix $X(\lambda)$ such that $N_{1}(\lambda)+$ $N_{2}(\lambda) X(\lambda)$ has least McMillan order.

The set of solutions to our problem can be written as

$$
\mathcal{S}(\lambda)=\left\{N_{1}(\lambda)+N_{2}(\lambda) X(\lambda) \mid \text { for all } X(\lambda)\right\}
$$

and the partitioned matrix $\left[N_{1}(\lambda) \quad N_{2}(\lambda)\right]$ is called a generator of $\mathcal{S}(\lambda)$. Note that by construction, each solution $Z(\lambda) \in \mathcal{S}(\lambda)$ satisfies the consistency condition $G^{T}(\lambda) Z(\lambda)=0$. To be a solution to the detector design problem, $Z(\lambda)$ must also satisfy $y_{i}(\lambda) Z(\lambda) \neq$ 0 , for $i=1, \ldots, q$, where $y_{i}(\lambda)$ is the $i$-th row of $Y(\lambda):=\left[\begin{array}{ll}G_{f}^{T}(\lambda) & 0\end{array}\right]$.

Assume $\left[N_{1}(\lambda) N_{2}(\lambda)\right]$ has the state space realization

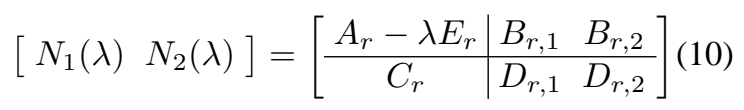

We have the following straightforward extension of the result of Morse (1976): 
Lemma 2. If $\left[N_{1}(\lambda) N_{2}(\lambda)\right]$ with state-space realization (10) generates $\mathcal{S}(\lambda)$, then so does $\left[\widehat{N}_{1}(\lambda) \widehat{N}_{2}(\lambda)\right]$ defined by

$\left[\widehat{N}_{1}(\lambda) \widehat{N}_{2}(\lambda)\right]=\left[\begin{array}{c|c}A_{r}+B_{r, 2} F_{r, 2}-\lambda E_{r} & B_{r, 1} B_{r, 2} \\ \hline C_{r}+D_{r, 2} F_{r, 2} & D_{r, 1} D_{r, 2}\end{array}\right]$

From Lemma 2 it follows that if $\left[\begin{array}{ll}N_{1}(\lambda) & N_{2}(\lambda)\end{array}\right]$ in (10) generates $\mathcal{S}(\lambda)$ then for any $F_{r, 2}$ and $L_{r}$, the system

$$
\left[\begin{array}{c|c}
A_{r}+B_{r, 2} F_{r, 2}-\lambda E_{r} & B_{r, 1}+B_{r, 2} L_{r} B_{r, 2} \\
\hline C_{r}+D_{r, 2} F_{r, 2} & D_{r, 1}+D_{r, 2} L_{r} D_{r, 2}
\end{array}\right]
$$

with TFM $\left[\widehat{N}_{1}(\lambda)+\widehat{N}_{2}(\lambda) L_{2}\right]$ generates $\mathcal{S}(\lambda)$ as well.

Using this result we can try to determine $L_{r}$ and $F_{r, 2}$ to achieve a pole-zero cancellation by making a maximum number of eigenvalues of $A_{r}+B_{r, 2} F_{r, 2}-$ $\lambda E_{r}$ uncontrollable via $B_{r, 1}+B_{r, 2} L_{r}$. This problem has been theoretically solved in (Morse, 1976). To apply the main result of (Morse, 1976) we need the following straightforward result:

Lemma 3. The system (11) is observable for all $F_{r, 2}$.

Morse (1976) has shown that a least order solution can be obtained by solving a minimal order dynamic cover design problem. Consider the set

$$
\mathcal{J}=\left\{\mathcal{V}: \operatorname{Im} \bar{B}_{r, 1}+\bar{A}_{r} \mathcal{V} \subset \operatorname{Im} \bar{B}_{r, 2}+\mathcal{V}\right\}
$$

where

$$
\left(\bar{A}_{r}, \bar{B}_{r, 1}, \bar{B}_{r, 2},\right):=\left(E_{r}^{-1} A_{r}, E_{r}^{-1} B_{r, 1}, E_{r}^{-1} B_{r, 2}\right)
$$

Let $\mathcal{J}^{*}$ denote the set of subspaces $\mathcal{J}$ of least dimension. If $\mathcal{V} \in \mathcal{J}^{*}$ then a pair $\left(F_{r, 2}, L_{r}\right)$ can be determined such that

$$
\left(\bar{A}_{r}+\bar{B}_{r, 2} F_{r, 2}\right) \mathcal{V}+\operatorname{Im}\left(\bar{B}_{r, 1}+\bar{B}_{r, 2} L_{r}\right) \subset \mathcal{V}
$$

Thus, determining a minimal dimension $\mathcal{V}$ is equivalent to a Type II minimal order cover design problem, and a conceptual approach to solve it has been indicated by Morse (1976). The outcome of his method is, besides $\mathcal{V}$, the pair $\left(F_{r, 2}, L_{r}\right)$ which achieves a maximal order reduction by pole-zero cancellations.

The approach suggested by Morse (1976) can be turned into a numerically reliable procedure. In what follows, due to space restrictions, we only sketch the proposed computational approach to determine $F_{r, 2}$ and $L_{r}$. The details of this procedure will be presented in another paper (Varga, 2003). The general idea is to perform a preliminary orthogonal similarity transformation on the system matrices in (10) by applying a special version of the controllability staircase form algorithm of Varga (1989) to the pair ([ $\left.B_{r, 2} B_{r, 1}\right], A_{r}-$ $\left.\lambda E_{r}\right)$. Since this pair is already in the controllability staircase form (8) and all structural information are already available, the computations do not involve any rank determinations and merely amount to update this form according to the desired column permutation of $B_{r}$. With additional block permutations and block row/column transformations, we can bring the transformed system matrices in the following special form

$$
\begin{aligned}
& Q\left(A_{r}-\lambda E_{r}\right) Z=\left[\begin{array}{c|c}
\widehat{A}_{11} & * \\
\hline \widehat{A}_{21} & \widehat{A}_{22}
\end{array}\right]-\lambda\left[\begin{array}{c|c}
\widehat{E}_{11} & * \\
\hline 0 & \widehat{E}_{22}
\end{array}\right] \\
& Q\left[B_{r, 2} B_{r, 1}\right]=\left[\begin{array}{cc}
0 & \widehat{B}_{12} \\
\hline \widehat{B}_{21} & \widehat{B}_{22}
\end{array}\right], \quad C_{r} Z=\left[\widehat{C}_{1} \mid \widehat{C}_{2}\right]
\end{aligned}
$$

where the pairs $\left(\widehat{B}_{12}, \widehat{A}_{11}-\lambda \widehat{E}_{11}\right)$ and $\left(\widehat{B}_{21}, \widehat{A}_{22}-\right.$ $\left.\lambda \widehat{E}_{22}\right)$ are controllable, and the submatrices $\widehat{B}_{21}, \widehat{B}_{22}$ and $\widehat{A}_{21}$ have the particular structure

$$
\left[\begin{array}{lll}
\widehat{B}_{21} & \widehat{B}_{22} & \widehat{A}_{21}
\end{array}\right]=\left[\begin{array}{ccc}
B_{21} & B_{22} & A_{21} \\
0 & 0 & 0
\end{array}\right]
$$

with $B_{21}$ having full row rank. Thus, by taking $L_{r}$ such that $B_{21} L_{r}+B_{22}=0$ and

$$
F_{r, 2}=\left[\begin{array}{cc}
\widehat{F}_{11} & 0 \\
0 & 0
\end{array}\right] Z^{-1}
$$

with $F_{11}$ satisfying $\widehat{B}_{21} \widehat{F}_{11}+\widehat{A}_{21}=0$, we achieve the cancellation of the maximum number of uncontrollable eigenvalues. The resulting system of least McMillan order is

$$
\left(\widehat{A}_{11}-\lambda \widehat{E}_{11}, \widehat{B}_{12}, \widehat{C}_{1}+D_{r, 2} \widehat{F}_{11}, D_{r, 1}+D_{r, 2} L_{r}\right)
$$

\section{NUMERICAL EXAMPLES}

The proposed method to compute rational nullspace bases has been implemented in a MATLAB $m$-function snull, based on the computation of orthogonal Kronecker-like forms available in the DESCRIPTOR Toolbox (Varga, 2000). The minimal cover algorithm of Varga (2003) has been implemented in the $m$-function smcover2, which relies on a special orthogonal controllability form algorithm (implemented as a Fortran 77 mex-file) and on a non-orthogonal reduction (implemented in MATLAB). The results presented in this section have been computed using the function $\mathrm{fd}$ implementing the proposed least order detector design procedure. Besides snull and smcover, $\mathrm{fd}$ also calls other tools of the DESCRIPTOR TOOLBOX as the minimal realization of generalized systems, finite-infinite/stable-unstable spectral separations, coprime factorization, etc.

To illustrate the applicability of the proposed approach to high order systems, we generated random standard continuous-time systems with state vector dimensions $n=5,10,20,40,80,160$ and fixed input and output vectors dimensions of $m_{d}=2, m_{f}=9, m_{d}=1$, 
$p=5$. For each system we determined a least order detector $R(s)$ which detects the occurrence of each of the 9 faults and decouples any influences from the command and disturbance inputs. To assess the effectiveness of the resulting detectors, we computed in each case the quantities

$$
\begin{aligned}
\eta_{f} & =\left\|R(s)\left[\begin{array}{c}
G_{f}(s) \\
O
\end{array}\right]\right\|_{\infty}>0, \\
\eta_{d, p} & =\left\|R(s)\left[\begin{array}{cc}
G_{d}(s) & G_{p}(s) \\
O & I
\end{array}\right]\right\|_{\infty}=0
\end{aligned}
$$

The results are summarized in Table 1 , where $n_{\text {detec }}$ is the order of the resulting detector. Note that for these randomly generated models the order of the rational nullspace is generically $n$, this being also the order of detectors computed using the methods of Patton and Hou (1998) and Hou (2000).

\begin{tabular}{cccc}
$n$ & $n_{\text {detec }}$ & $\eta_{f}$ & $\eta_{d, p}$ \\
\hline 5 & 1 & 2.21 & $7.70 \cdot 10^{-16}$ \\
10 & 2 & 1.45 & $1.31 \cdot 10^{-15}$ \\
20 & 5 & 6.62 & $2.31 \cdot 10^{-14}$ \\
40 & 10 & 4.64 & $3.75 \cdot 10^{-14}$ \\
80 & 20 & 4.80 & $3.01 \cdot 10^{-11}$ \\
160 & 40 & 2,326 & $3.13 \cdot 10^{-7}$
\end{tabular}

Table 1. Computational results

\section{CONCLUSION}

We proposed a numerically sound computational approach to design least order fault detectors. The involved main computational ingredients are: (1) the computation of a rational nullspace basis of a rational matrix; (2) the reduction of the dynamical order of the detector. For the computation of rational nullspace bases, a new numerically stable algorithm, based on orthogonal pencil reduction, has been proposed. For the order reduction of the detector, a method has been proposed able to determine least order detectors starting from a given generator of all solutions. This method is based on determining least order dynamical covers using methods derived from computation of controllability staircase forms (Varga, 2003). We believe that the overall approach to design least order detectors is a viable alternative to polynomial bases based approaches. Numerical examples illustrate the potential of this approach to handle in a numerically reliable way large order systems.

\section{REFERENCES}

Beelen, Th.G.J. (1987). New Algorithms for Computing the Kronecker structure of a Pencil with Applications to Systems and Control Theory. $\mathrm{Ph}$. D. Thesis. Eindhoven University of Technology.

Benner, P., V. Mehrmann, V. Sima, S. Van Huffel and A. Varga (1999). SLICOT - a subroutine library in systems and control theory. In: $A p$ plied and Computational Control, Signals and
Circuits (B. N. Datta, Ed.), Vol. 1., pp. 499-539. Birkhäuser.

Chen, J. and R. J. Patton (1999). Robust Model-Based Fault Diagnosis for Dynamic Systems. Kluwer Academic Publisheres, London.

Forney, G. D. (1975). Minimal bases of rational vector spaces with applications to multivariable linear systems. SIAM J. Control 13, 493-520.

Frisk, E. (2000). Order of residual generators - bounds and algorithms. Prepr. IFAC Symp. SAFEPROCESS'2000, Budapest, Hungary. pp. 599-604.

Frisk, E. and M. Nyberg (2001). A minimal polynomial basis solution to residual generation for fault diagnosis in linear systems. Automatica 37, 1417-1424.

Gertler, J. (1998). Fault Detection and Diagnosis in Engineering Systems. Marcel Dekker. New York.

Hou, M. (2000). Fault detection and isolation for descriptor systems. In: Issues of Fault Dyagnosis for Dynamic Systems (R. J. Patton, P. M. Frank and R. N. Clark, Eds.), Springer Verlag, London, pp. $115-144$.

Kailath, T. (1980). Linear Systems. Prentice Hall, Englewood Cliffs, N.J.

Morse, A. S. (1976). Minimal solutions to transfer matrix equations. IEEE Trans. Autom. Control 21, 131-133.

Patton, R. J. and M. Hou (1998). Design of fault detection and isolation observers: a matrix pencil approach. Automatica 34, 1135-1140.

Van Dooren, P. (1981). The generalized eigenstructure problem in linear systems theory. IEEE Trans. Autom. Control 26, 111-129.

Varga, A. (1989). Computation of irreducible generalized state-space realizations. Kybernetika 26, 89106.

Varga, A. (1996). Computation of Kronecker-like forms of a system pencil: Applications, algorithms and software. Proc. CACSD'96 Symposium, Dearborn, MI. pp. 77-82.

Varga, A. (1998). Computation of coprime factorizations of rational matrices. Lin. Alg. \& Appl. 271, 83-115.

Varga, A. (2000). A descriptor systems toolbox for Matlab. Proc. CACSD'2000 Symposium, Anchorage, Alaska.

Varga, A. (2002). Computational issues in faultdetection filter design. Proc. of CDC'2002, Las Vegas, Nevada.

Varga, A. (2003). Reliable algorithms for computing minimal dynamic covers. (submitted to CDC'2003)

Verghese, G., P. Van Dooren and T. Kailath (1979). Properties of the system matrix of a generalized state-space system. Int. J. Control 30, 235-243.

Wang, S.-H. and E. J. Davison (1973). A minimization algorithm for the design of linear multivariable systems. IEEE Trans. Autom. Control 18, 220225 . 